TITLE:

\title{
Impaired overt facial mimicry in response to dynamic facial expressions in high-functioning autism spectrum disorders.
}

\section{AUTHOR(S):}

Yoshimura, Sayaka; Sato, Wataru; Uono, Shota; Toichi, Motomi

\section{CITATION:}

Yoshimura, Sayaka ...[et al]. Impaired overt facial mimicry in response to dynamic facial expressions in high-functioning autism spectrum disorders.. Journal of autism and developmental disorders 2015, 45(5): 1318-1328

ISSUE DATE:

2015-05-01

URL:

http://hdl.handle.net/2433/197725

\section{RIGHT:}

The final publication is available at Springer via http://dx.doi.org/10.1007/s10803-0142291-7; 許諾条件により本文ファイルは2016-05-01に公開.; This is not the published version. Please cite only the published version.; この論文は出版社版でありません。引用 の際には出版社版をご確認ご利用ください。 
Impaired overt facial mimicry in ASD

\section{Abstract}

Previous electromyographic studies have reported that individuals with autism spectrum disorders (ASD) exhibited atypical patterns of facial muscle activity in response to facial expression stimuli. However, whether such muscle activity is expressed in visible facial mimicry remains unknown. To investigate this issue, we videotaped facial responses in high-functioning individuals with milder ASD symptoms and typically developing controls to dynamic and static facial expressions of anger and happiness. Visual coding of facial muscle activity and the subjective impression ratings consistently showed reduced congruent responses to dynamic expressions in the ASD group. Additionally, this decline in congruent facial reactions was related to social dysfunction. These results suggest that impairment in the ability to demonstrate overt facial mimicry in response to others' dynamic facial expressions may underlie difficulties in reciprocal social interaction among individuals with ASD.

\section{Keywords}

Autism spectrum disorders (ASD); dynamic facial expression; facial mimicry, reciprocal social interaction 
Impaired overt facial mimicry in ASD

\section{Introduction}

Impairment in reciprocal social interactions is one of the core features of autism spectrum disorders (ASD) (American Psychiatric Association 2000). The diagnostic criteria for ASD include impairment in the use of nonverbal behaviors, including facial expressions, to regulate social interactions.

In accord with these criteria, impairment in facial mimicry during social interactions has often been reported in individuals with ASD (Yirmiya et al. 1989; Stel et al. 2008). Facial mimicry is the spontaneous production of a facial expression that is congruent with an observed facial expression (Hess and Blairy 2001). This behavior affects recognition of emotion in response to others' facial expressions (Künecke et al. 2014; Sato et al. 2013) and facilitates reciprocal social interaction, such as the establishment of rapport and the development of empathy (Hess et al. 1999). For example, mimicking an interaction partner's smile tends to increase his/her like of the mimicker (Cappella 1993). Previous studies have reported that individuals with ASD displayed reduced and/or inappropriate facial reactions compared with typically developing individuals in response to realistic social stimuli such as the facial expressions of other individuals in social interactions (Yirmiya et al. 
Impaired overt facial mimicry in ASD

1989) and the emotional expressions of a character in a video (Stel et al. 2008).

However, these studies under realistic conditions assessed factors other than facial mimicry. In realistic interactions, facial expressions, environmental stimuli other than facial expressions, and the contexts in which social interactions occur provide social information (e.g., the context of conversation provides information about synchronous laughing). Because these environmental stimuli and contexts also induce responses in the sender and receiver of social information in reciprocal interactions (e.g., Goodwin 2002; Nakano et al. 2009), realistic studies cannot exclude the possibility that factors other than facial expressions influence facial actions.

Studies recording facial electromyography (EMG) under controlled conditions have been used to overcome the limitations of realistic studies (McIntosh et al. 2006; Beall et al. 2008; Oberman et al. 2009). EMG studies have removed stimuli other than facial expressions and presented expressions within a very short time to exclude the effects of context. Therefore, these studies examined facial reactions to others' facial expressions per se. The results of these EMG studies revealed that individuals with ASD had reduced (McIntosh et al. 2006), delayed, 
Impaired overt facial mimicry in ASD

(Oberman et al. 2009), or atypical (Beall et al. 2008) facial muscle activity in response to the photographs of emotional facial expressions. These studies suggest that individuals with ASD are impaired in terms of facial mimicry.

However, EMG studies are limited in that it is unclear whether the facial muscle activity revealed by EMG is externally observable as facial mimicry. Because facial EMG amplitude changes are very subtle (a few microvolts; e.g., Sato et al. 2008), facial muscle activity may not be visible (Cacioppo et al. 1986; Filipo et al. 2002; De Seta et al. 2011); hence, the difference in EMG data between typically developing and ASD groups may not be evident in overt facial mimicry. This point is crucial, because impairments in overt facial mimicry can produce problems in reciprocal social interaction, leading to deficiencies in reciprocal social interaction, such as difficulty establishing rapport with partners. Therefore, further studies are needed to examine whether individuals with ASD actually demonstrate observable facial mimicry.

The use of a dynamic or static presentation condition may be relevant in investigating this issue. Dynamic facial expressions are more natural and powerful as social stimuli than are static expressions. Previous studies (Weyers et al. 2006; Sato and Yoshikawa 2007; Sato et al. 2008) 
Impaired overt facial mimicry in ASD

have shown that dynamic facial expressions had facilitative effects on facial mimicry compared with static ones. Functional neuroimaging studies have also found that typically developing participants exhibited increased activation in brain regions that are thought to be the neural basis of facial mimicry (Likowski et al. 2012) in response to dynamic compared with static facial expressions (e.g., Kilts et al. 2003; LaBar et al. 2003; Sato et al. 2004). In contrast, some studies have suggested that dynamic presentations of facial stimuli elicit atypical behavioral patterns in individuals with ASD more clearly than do static presentations (Kessels et al. 2010; Tardif et al. 2007; Uono et al. 2009). Functional neuroimaging studies have demonstrated that individuals with ASD, compared with typical developing individuals, exhibited markedly reduced activation in the social brain network in response to dynamic versus static facial expressions (Pelphrey et al. 2007; Sato et al. 2012). Based on these data, we hypothesized that individuals with ASD would exhibit apparent impairment in overt facial mimicry in response to dynamic facial expressions.

The current study examined overt facial mimicry in response to dynamic and static facial expressions of anger and happiness in high-functioning individuals with milder ASD symptoms and in typically 
Impaired overt facial mimicry in ASD

developing age-, sex-, and IQ-matched controls. We videotaped the facial movements of participants during their passive observation of emotional facial expressions. We coded the overt facial muscle movements (Ekman and Friesen 1978) and evaluated the subjective emotional impressions (Kring and Sloan 2007) conveyed by the facial reactions of participants. We also examined intentional imitations of facial expressions to confirm that the impairment in facial mimicry was not caused by problems in peripheral facial muscle activity. Furthermore, we evaluated the degree of social dysfunction in individuals with ASD and tested the relationship between overt facial reactions and social dysfunction. We predicted that ASD participants would exhibit reduced overt activity of the facial muscles and reduced emotional expression congruent with the presented dynamic facial expressions compared with typically developing participants. We also predicted that the reduced facial reactions to dynamic facial expressions would be related to social dysfunction in individuals with ASD.

\section{Materials and methods}

\section{Participants}

The ASD group was composed of 15 adults (12 males, three females; 
Impaired overt facial mimicry in ASD

age: $M=26.2 \mathrm{yrs}, S D=6.9 \mathrm{yrs})$. Although an additional female candidate participated, her data were not analyzed due to the high frequency of artifacts (over $15.6 \%$ of all trials). The group included six males with Asperger's disorder and nine (three females, six males) with pervasive developmental disorder not otherwise specified (PDD-NOS). As defined in the Diagnostic and Statistical Manual-Fourth Edition-Text Revision (DSM-IV-TR) (American Psychiatric Association 2000), PDD-NOS includes heterogeneous subtypes of ASD, ranging from so-called atypical autism to a subgroup with symptoms milder than Asperger's disorder (i.e., satisfying fewer diagnostic criteria than required for a diagnosis of Asperger's disorder). In this study, only high-functioning participants with PDD-NOS with milder symptoms than those associated with Asperger's disorder were included. Neurological and psychiatric problems other than those associated with ASD were ruled out (i.e., ASD participants had no comorbid psychiatric conditions such as major depressive or anxiety disorders). No participants were taking medication. Therefore, all participants in the ASD group had only the core deficits of ASD (i.e., reciprocal social impairments and restricted, repetitive traits).

The diagnosis was made based on DSM-IV-TR using a stringent procedure in which every item of the ASD diagnostic criteria was 
Impaired overt facial mimicry in ASD

investigated in interviews with participants and their parents (and professionals who helped them, if any) conducted by two psychiatrists with expertise in developmental disorders. Only participants who had at least one of four social impairments (i.e., impairment in nonverbal communication including lack of joint attention, sharing interests, relationships with peers, and emotional and interpersonal mutuality) and did not satisfy any of the criteria for autistic disorder, such as language delay, were included. For diagnostic purposes, comprehensive interviews were conducted to obtain information about participants' developmental histories.

Symptom severity of ASD participants was assessed using the Childhood Autism Rating Scale-Tokyo version (Kurita et al. 1989), which is the Japanese version of the Childhood Autism Rating Scale (CARS) (Schopler et al. 1986). Two psychiatrists who made the ASD diagnoses administered the CARS. The CARS includes 15 items that assess autism-related behaviors. Each item is scored from 1.0 to 4.0 in units of 0.5, with higher scores indicating more severe impairment. The total CARS score, which is the sum of all items, can range from 15.0 to 60.0. The total scores of most ASD participants were below the cut-off score of 30.5 for a diagnosis of autistic disorder (cf. Tachimori et al. 2003). The 
Impaired overt facial mimicry in ASD

CARS score of participants in the present study $(M=23.87, S D=3.44)$ was comparable to those of individuals with high-functioning ASD, as reported by Koyama et al. (2007).

Intelligence quotients (IQs), measured by the Wechsler Adult Intelligence Scale-Third edition (WAIS-III), fell within the normal range for all participants in the ASD group (verbal IQ (VIQ): $M=112.7, S D=$ 18.3; performance IQ (PIQ): $M=110.1, S D=16.8)$.

The control group was composed of 15 adults (nine males, six females; age, $M=24.1, S D=4.0$ ). They had no neurological or psychiatric problems. The WAIS-III IQs of all control participants also fell within the normal range (VIQ: $M=120.1, S D=10.0$; PIQ: $M=116.6$, $S D=11.3)$. They were recruited through advertisements and were matched with the ASD group for age, $t(28)=-1.01, p=.32, r=.19$, sex, Fisher's exact test, $p=.43, \varphi=.22$, VIQ, $t(28)=1.61, p=.12, r=.29$, and $\mathrm{PIQ}, t(28)=1.28, p=.21, r=.24$.

All participants had normal or corrected-to-normal visual acuity. All participants were right-handed, as assessed by the Edinburgh Handedness Inventory (Oldfield 1971). Ethical approval for this study was obtained from the Ethics Committee of Kyoto University Graduate School and Faculty of Medicine. Written informed consent was obtained from all 
Impaired overt facial mimicry in ASD

participants.

\section{Apparatus}

Experimental events were controlled by a program written in Visual $\mathrm{C}++5.0$ and implemented on a computer (Inspiron 8000 , Dell) with a Microsoft Windows operating system.

We used a video prompter system (MPL-20, Life-On) to present stimuli and unobtrusively videotaped participants' facial reactions. The system consisted of a 20-inch monitor, a two-way mirror, and other interfaces. The monitor was tilted up (the participants could not see it because of a curtain), and the screen was reflected onto a two-way mirror mounted at a $45^{\circ}$ angle to the screen. The mirror reflected the screen, creating a viewing situation similar to that of an ordinary 20 -inch monitor. The distance between the mirror and the participants' eyes was approximately $1.9 \mathrm{~m}$. The monitor screen was set to a resolution of 600 vertical $\times 800$ horizontal pixels, 16-bit color, and a frame refresh rate of $75 \mathrm{~Hz}$. The stimuli were presented at 300 vertical $\times 200$ horizontal pixels and subtended a visual angle of about $5.4^{\circ}$ vertically $\times 3.8^{\circ}$ horizontally. The background was held constant with a gray color.

A video camera (HDR-CX700V, Sony) was placed behind the 
Impaired overt facial mimicry in ASD

two-way mirror. The video camera recorded participants' full faces at a rate of 30 frames per second (i.e., $33.3 \mathrm{~Hz}$ ). The video data were automatically acquired using a video data recorder (AQ-VU, Teac) at a rate of 30 frames per second.

\section{Stimuli}

The stimuli were identical to those used in a previous study (Experiment 2 in Sato and Yoshikawa 2007). Materials were full-color video clips of angry and happy facial expressions of eight females and eight males. These stimuli were selected from a video database of facial expressions of emotion portrayed by more than 50 Japanese models. None of the faces was familiar to any of the participants. Preliminary ratings from 14 individuals who did not participate in this experiment confirmed that the stimuli clearly displayed the target emotions relative to other basic emotions. Additionally, a coder trained in the Facial Action Coding System (FACS) (Ekman and Friesen 1978) evaluated the stimulus facial actions, and the FACS data were interpreted by reference to the Facial Action Coding System Affect Interpretation Dictionary (Ekman et al. 1998). The results confirmed that the emotional meanings of the stimulus facial actions could be recognized 
Impaired overt facial mimicry in ASD

as intended. Specifically, all the selected angry and happy expressions involved action units (AUs) 4 (brow lowering) and 12 (lip-corner pulling), respectively. The expressions contained few artifacts irrelevant to emotional expressions.

In terms of the dynamic expression stimuli, 38 frames representing transitions from neutral to emotional expressions were presented. Each frame was shown for $40 \mathrm{~ms}$, and each clip lasted for a total of 1,520 $\mathrm{ms}$.

The frames showing the apex emotional expressions under the dynamic condition were used as the static expressions and presented for $1520 \mathrm{~ms}$.

\section{Procedure}

Experiments were conducted individually in a sound-proof room. Upon arrival, participants were told that the experiment concerned the evaluation of faces. Subsequently, they were told that they would be watching some facial stimuli and later answering some questions about the faces after watching them again and that the method for evaluation would be presented after they had watched a complete set of facial stimuli. Therefore, they did not need to answer questions about facial stimuli in the first (i.e., facial mimicry) task. Participants relaxed for about 10 
Impaired overt facial mimicry in ASD

minutes to allow general adaptation to the experimental room, and then they viewed instructions displayed on the monitor explaining the stimulus presentation.

Participants took part in a total of 32 trials of the facial mimicry task (eight trials each of dynamic angry, dynamic happy, static angry, and static happy expressions). The order of stimulus presentation was randomized. All participants engaged in a few practice trials to familiarize themselves with the stimulus presentation before data collection. To avoid habituation and drowsiness, participants had a short rest period halfway through the trials. Throughout the stimulus presentations, participants' full faces were continuously videotaped by the video camera behind the screen; participants were not aware of the videotaping.

In each trial, a fixation point (a picture with a small “+” presented in gray on a white background that was the same size as the stimuli) was presented at the center of the screen for $1,520 \mathrm{~ms}$. Then, the stimulus was presented for $1,520 \mathrm{~ms}$. After stimulus presentation, the screen was filled with a gray color during the inter-trial interval, which was controlled to vary randomly from 6,000 to $9,000 \mathrm{~ms}$. Throughout data acquisition, 
Impaired overt facial mimicry in ASD

participants' motion artifacts were monitored through a video monitor, and the stimulus presentations were suspended when participants exhibited unrelated facial movements.

Following video recording of 32 trials, participants were given instructions for evaluating the facial stimuli. Then, they were asked to evaluate the affective valence and arousal level of facial stimuli after watching them again. We do not report the results of these evaluations here, as our objective in the present study was to evaluate overt facial mimicry.

After evaluating the facial stimuli, participants performed a total of 16 trials of an intentional imitation task (four trials each of dynamic angry, dynamic happy, static angry, and static happy expressions). The procedure was almost the same as the facial mimicry task except for the instruction. Before the beginning of the task, participants were instructed to imitate the facial expression depicted in every stimulus after the stimulus disappeared from the monitor; participants were told that this task was designed to clarify whether imitating facial expressions would affect the evaluation of faces.

Finally, participants were interviewed to determine whether they had been aware that their faces had been videotaped. This process confirmed 
Impaired overt facial mimicry in ASD

that all participants had been unaware of the videotaping. Participant permission to use the videotapes for analysis was requested and granted in all cases. Additional permission was requested from participants whose images are used here as examples, and written consent was obtained.

Data analysis

Preprocessing of video data. Video data were processed using AQView (Teac). A 3500-ms sample consisting of $1000 \mathrm{~ms}$ of pre-stimulus baseline data (presentation of the fixation point) and $2500 \mathrm{~ms}$ of data collected after stimulus onset was taken from each trial. The time window of the post-stimulus period was identical to that in a previous behavioral study (Sato and Yoshikawa 2007) that detected facial mimicry in response to dynamic facial expressions.

Because participants were not told that their faces were being videotaped, the data included various types of artifacts (e.g., mumbling). One of the authors, who was blind to the condition under which the tapes were made, checked the videotapes and rejected the artifact-contaminated trials. To evaluate the artifacts in the video data, we used a slightly modified version of the artifact lists employed in a previous study (Sato and Yoshikawa 2007). The artifact lists included resting the chin on the 
Impaired overt facial mimicry in ASD

hand (this prevented movement of the lower face), swinging the head, yawning, mumbling, and displaying facial expressions during the pre-stimulus period. The mean numbers of artifact-contaminated trials in the facial mimicry task $( \pm S D)$ for dynamic anger, dynamic happiness, static anger, and static happiness were $0.7 \pm 0.9,0.8 \pm 1.0,0.5 \pm 0.7$, and $0.3 \pm 0.6$ in the ASD group and $0.6 \pm 0.7,0.7 \pm 0.7,0.6 \pm 0.8$, and $0.4 \pm 0.5$ in the control group, respectively. Those in the intentional imitation task $( \pm S D)$ were $0.1 \pm 0.3,0.1 \pm 0.3,0.1 \pm 0.3$, and $0.0 \pm 0.0$ in the ASD group and $0.0 \pm 0.0,0.8 \pm 1.0,0.0 \pm 0.0$, and $0.0 \pm 0.0$ in the control group, respectively. Three-way repeated-measures analyses of variance (ANOVA) with group as a between-participants factor and presentation condition and expression as within-participants factors revealed no significant main effects or interactions on the facial mimicry task, $F(1,28)$ $<2.28, p \mathrm{~s}>.10, \eta_{\mathrm{p}}{ }^{2}<.08$, or on the intentional imitation task, $F(1,28)<$ $2.01, p \mathrm{~s}>.10, \eta_{\mathrm{p}}{ }^{2}<.07$, indicating that none of the factors influenced the number of artifact-contaminated trials. The artifact-contaminated data were eliminated from subsequent analyses.

FACS coding. Participants' facial reactions in response to each stimulus were coded using FACS (Ekman and Friesen 1978), a comprehensive, anatomically based coding system that describes visible 
Impaired overt facial mimicry in ASD

facial muscle movements in terms of AUs; coders do not make interpretations. Based on our research interests, only AU 4 (brow lowering, a prototypical facial action in angry expressions) and AU 12 (lip-corner pulling, a prototypical facial action in happy expressions) were evaluated.

A scorer trained in FACS coding who was blind to the experimental protocol scored the facial reactions exhibited by participants within the $2,500 \mathrm{~ms}$ after stimulus onset. To confirm the reliability of the coding, another scorer independently coded the data from four participants in each group (i.e., 26.7\%). Inter-scorer reliabilities were found to be sufficiently high (Kappa coefficient $=.90$ and .76 for AU 4 and AU12, respectively) .

Data on the frequency of each AU were analyzed using three-way repeated-measures ANOVAs. Group was treated as a between-participants factor, and presentation condition and expression were treated as within-participant factors. The ASD subgroups (Asperger's disorder and PDD-NOS) were integrated as the ASD group, as preliminary analyses revealed no significant main effect of diagnosis and no interaction of diagnosis with presentation condition or expression in terms of either AU, $F(2,27)<2.60, p \mathrm{~s}>.05, \eta_{\mathrm{p}}{ }^{2}<.17$. Follow-up simple-effect analyses were conducted for significant interactions (cf. Kirk 1995). When higher-order 
Impaired overt facial mimicry in ASD

interactions were significant, the main effects or lower-order interactions were not subjected to interpretation (cf. Tabachnick and Fidell 2001).

The results of all tests in this report were considered statistically significant at $p<.05$, unless stated otherwise.

FACES coding. The participants' facial reaction in response to each stimulus was also coded using the Facial Expression Coding System (FACES) (Kring and Sloan 2007). FACES is a rating system used to capture the valence (negative, positive) of expressive facial behavior. A scorer trained in FACES coding who was blind to the experimental protocol scored the facial reactions exhibited by the participants within the $2,500 \mathrm{~ms}$ after stimulus onset. To confirm the reliability of coding, another scorer independently coded data from four participants in each group (i.e., 26.67\%). Inter-scorer reliabilities were found to be sufficiently high (Kappa coefficient $=.82$ and .72 for positively and negatively valenced data, respectively).

The frequency data for each valence were separately analyzed using ANOVAs of the same design as those used in the FACS analysis.

Coding of attention. Coding of attention was conducted to evaluate whether attentional problems affected facial responses. The facial reaction coder rated participants' attention to stimuli according to the 
Impaired overt facial mimicry in ASD

procedure described in a previous study (Stel et al. 2008). The coder scored the number of times participants failed to look at a stimulus during the presentation. Then, the average inattention scores per stimulus were analyzed using ANOVAs of the same design as those used for FACS and FACES.

Relationship between the frequency of facial mimicry and ASD symptom severity. Pearson's correlation coefficients were calculated for the ASD group to test the relationship between the frequency of facial mimicry and the severity of social dysfunction. We calculated the average score of AU4 occurrences in response to an angry expression and AU12 occurrences in response to a happy expression for each presentation condition (i.e., dynamic and static conditions) during the facial mimicry task, and we used these as the measure of the frequency of facial mimicry evaluated by FACS under dynamic and static conditions, respectively. We also calculated the frequency of facial mimicry evaluated by FACES in the same way. Following Uono et al. (2011), we used the sum of five CARS items that were commonly categorized into the social functioning construct in previous studies evaluating the factor structure of the CARS (cf. Magyar and Pandolfi 2007) as the social dysfunction index. We excluded multivariate outliers by calculating the Mahalanobis distance for 
Impaired overt facial mimicry in ASD

each $(p<.05)$. The significance of the correlation coefficients was evaluated using $t$-statistics (one-tailed). The relationships between facial mimicry and non-social dysfunction (i.e., stereotypies and sensory abnormality), that is, the sum of three CARS items (cf. Magyar and Pandolfi, 2007), and between intentional imitation and social dysfunction were also examined using two-tailed $t$-statistics, although we did not have specific hypotheses for these relationships.

\section{Results}

Facial mimicry

Figure 1 presents representative examples of participants' facial reactions.

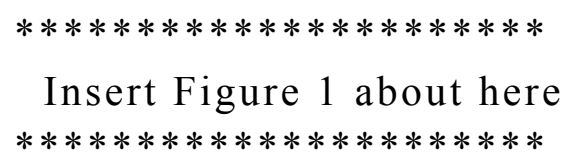

FACS data. With regard to AU 4 (Fig. 2(a), left), the highest-order three-way interaction among group, presentation condition, and expression was significant, $F(1,28)=4.23, p=.049, \eta_{\mathrm{p}}{ }^{2}=.13$. Additionally, the main effect of expression was significant, $F(1,28)=$ $12.18, p=.002, \eta_{\mathrm{p}}^{2}=.30$.

Insert Figure 2 about here 
Impaired overt facial mimicry in ASD

Based on our research objectives, we conducted two follow-up analyses of the three-way interactions. First, to investigate the differences in facial actions under each condition, we tested the simple-simple main effect of expression. The results revealed that the effect of expression was significant under the dynamic, $F(1,56)=20.44, p$ $<.001, \eta_{\mathrm{p}}{ }^{2}=.27$, and static, $F(1,56)=5.95, p=.018, \eta_{\mathrm{p}}{ }^{2}=.10$ presentation conditions in the control group, revealing more frequent AU 4 responses to angry than to happy expressions in the control group. The simple-simple main effect of expression was not significant under the dynamic and static presentation conditions in the ASD group, $F(1,56)<$ $1.43, p \mathrm{~s}>.10, \eta_{\mathrm{p}}{ }^{2}<.03$. Next, to test group differences under each condition, we analyzed the simple-simple main effect of group. The effect of group was significant only for angry expressions under the dynamic presentation condition, $F(1,112)=9.95, p=.002, \eta_{\mathrm{p}}{ }^{2}=.08$, indicating more frequent AU 4 responses to dynamic angry expressions in the control group than in the ASD group.

In terms of $\mathrm{AU} 12$ (Fig. 2(a), right), the three-way interaction was significant, $F(1,28)=4.66, p=.040, \eta_{\mathrm{p}}{ }^{2}=.14$. The main effect of expression, $F(1,28)=10.44, p=.003, \eta_{\mathrm{p}}{ }^{2}=.27$, and the interaction 
Impaired overt facial mimicry in ASD

between group and expression, $F(1,28)=4.84, p=.036, \eta_{\mathrm{p}}{ }^{2}=.15$, were also significant.

Follow-up analyses were conducted for the three-way interaction as in the case of AU 4. The simple-simple main effect of expression was significant only under the dynamic presentation condition in the control group, $F(1,56)=18.82, p<.001, \eta_{\mathrm{p}}{ }^{2}=.25$, indicating more frequent AU 12 responses to dynamic happy versus angry expressions in the control group. In the ASD group, the simple-simple main effect of expression was not significant under the dynamic and static presentation conditions, $F(1,56)<1.26, p \mathrm{~s}>.10, \eta_{\mathrm{p}}{ }^{2}<.03$. A significant simple-simple main effect of group was also found only for happy expressions under the dynamic presentation condition, $F(1,112)=4.61, p=.034, \eta_{\mathrm{p}}{ }^{2}=.04$, indicating more frequent AU 12 responses to dynamic happy expressions in the control group than in the ASD group.

FACES data. With regard to negatively valenced responses (Fig. 2(b), left), the highest-order three-way interaction among group, presentation condition, and expression was significant, $F(1,28)=4.37, p=.046, \eta_{\mathrm{p}}{ }^{2}$ $=.13$. Additionally, the main effect of expression, $F(1,28)=16.89, p$ $<.001, \eta_{\mathrm{p}}{ }^{2}=.38$, the interaction between group and presentation condition, $F(1,28)=7.27, p=.011, \eta_{\mathrm{p}}{ }^{2}=.21$, and the interaction between 
Impaired overt facial mimicry in ASD

group and expression, $F(1,28)=4.23, p=.049, \eta_{\mathrm{p}}{ }^{2}=.13$, were significant.

Follow-up analyses for the three-way interaction were conducted in the same way as with the FACS analyses. The simple-simple main effect of expression was significant under the dynamic, $F(1,56)=22.06, p$ $<.001, \eta_{\mathrm{p}}{ }^{2}=.28$, and static, $F(1,56)=7.45, p=.009, \eta_{\mathrm{p}}{ }^{2}=.12$, presentation conditions in the control group, indicating more frequent negatively valenced responses to angry expressions than to happy expressions in the control group. In the ASD group, the simple-simple main effect of expression was not significant under the dynamic and static presentation conditions, $F(1,56)<3.25, p \mathrm{~s}>.05, \eta_{\mathrm{p}}{ }^{2}<0.06$. The simple-simple main effect of group was significant only for angry expressions under the dynamic presentation condition, $F(1,112)=9.32, p$ $=.003, \eta_{\mathrm{p}}{ }^{2}=.08$, indicating more frequent negatively valenced responses to dynamic angry expressions in the control group than in the ASD group. In terms of positively valenced responses (Fig. 2(b), right), the three-way interaction showed a non-significant trend, $F(1,28)=3.34, p$ $=.078, \eta_{\mathrm{p}}{ }^{2}=.11$. The main effect of expression, $F(1,28)=15.25, p<.001$, $\eta_{\mathrm{p}}{ }^{2}=.35$, and the interaction between group and presentation condition, $F(1,28)=4.21, p=.05, \eta_{\mathrm{p}}^{2}=.13$, were significant. 
Impaired overt facial mimicry in ASD

Based on our research objectives, we conducted follow-up analyses for the three-way interaction in the same manner as with negatively valenced responses. The simple-simple main effect of expression was significant under the dynamic, $F(1,56)=20.85, p<.001, \eta_{\mathrm{p}}{ }^{2}=.27$, and static, $F(1,56)=5.35, p=.024, \eta_{\mathrm{p}}{ }^{2}=.09$, presentation conditions in the control group, indicating more frequent positively valenced responses to happy than to angry expressions in the control group. This effect was not significant under the dynamic and static presentation conditions in the ASD group, $F(1,56)<2.11, p \mathrm{~s}>.10, \eta_{\mathrm{p}}{ }^{2}<.04$. The analyses also revealed a significant simple-simple main effect of group only for happy expressions under the dynamic presentation condition, $F(1,112)=8.85, p$ $=.004, \eta_{\mathrm{p}}{ }^{2}=.07$, indicating more frequent positively valenced responses to dynamic happy expressions in the control group than in the ASD group.

Intentional imitation of facial expression.

FACS data. With regard to AU 4 (Fig. 3(a), left), only the main effect of expression was significant, $F(1,28)=665.36, p<.001, \eta_{\mathrm{p}}{ }^{2}=.96$, indicating that only expression influenced the production of AU4 responses.

\footnotetext{
$* * * * * * * * * * * * * * * * * * * * * *$

Insert Figure 3 about here
} 
Impaired overt facial mimicry in ASD

Also in terms of AU 12 (Fig. 3(a), right), only the main effect of expression was significant, $F(1,28)=657.74, p<.001, \eta_{\mathrm{p}}{ }^{2}=.96$, indicating that only expression influenced the production of AU12 responses.

FACES data. With regard to negatively valenced responses (Fig. 3(b), left), the main effect of expression, $F(1,28)=362.93, p<.001, \eta_{\mathrm{p}}{ }^{2}=.93$, and the interaction between group and expression, $F(1,28)=5.09, p$ $=.032, \eta_{\mathrm{p}}^{2}=.15$ were significant .

Follow-up analyses were conducted for the two-way interaction. The results revealed that the simple main effect of expression was significant in both the control group, $F(1,28)=226.99, p<.001, \eta_{\mathrm{p}}{ }^{2}=.80$, and the ASD group, $F(1,28)=141.03, p<.001, \eta_{\mathrm{p}}{ }^{2}=.72$, indicating that negatively valenced responses occurred more frequently in response to angry expressions than to happy expressions in both groups. The simple main effect of group was not significant for either angry expressions or happy expressions, $F(1,56)<3.49, p \mathrm{~s}>.05, \eta_{\mathrm{p}}{ }^{2}<.06$.

Regarding positively valenced responses (Fig. 3(b), right), only the main effect of expression was significant, $F(1,28)=330.30, p<.001, \eta_{\mathrm{p}}{ }^{2}$ $=.92$, indicating that only expression influenced the production of 
Impaired overt facial mimicry in ASD

positively valenced responses.

In summary, in contrast to the results for spontaneous facial mimicry, no significant three-way interactions involving either the FACS or the FACES measures were observed, indicating that the ASD group did not evidently differ from the control group in their intentional facial imitations in response to dynamic facial expressions.

\section{Attention.}

The average inattention score per stimulus in response to dynamic angry, dynamic happy, static angry, and static happy stimuli in the facial mimicry task $( \pm S D)$ was $0.04 \pm 0.09,0.01 \pm 0.04,0.05 \pm 0.09$, and $0.01 \pm$ 0.03 in the ASD group, and $0.05 \pm 0.08,0.05 \pm 0.10,0.03 \pm 0.06$, and 0.05 \pm 0.07 in the control group, respectively. The main effects of group and experimental condition and the interaction between group and experimental condition all failed to reach significance, $F(1,28)<3.27$, $p$ s $>.05, \eta_{\mathrm{p}}{ }^{2}<.10$. The average inattention score per stimulus in response to dynamic angry, dynamic happy, static angry, and static happy stimuli in the intentional imitation task $( \pm S D)$ was $0.04 \pm 0.10,0.06 \pm 0.11,0.01 \pm$ 0.05 , and $0.03 \pm 0.13$ in the ASD group and $0.02 \pm 0.06,0.01 \pm 0.04,0.01$ \pm 0.05 , and $0.00 \pm 0.00$ in the control group, respectively. The ANOVA 
Impaired overt facial mimicry in ASD

revealed no significant main effect or interaction, $F(1,28)<2.33, p \mathrm{~s}>.10$, $\eta_{\mathrm{p}}^{2}<.08$

The relationship between the frequency of facial mimicry and ASD symptom severity

Correlation analyses revealed that the correlation between the frequency of facial mimicry evaluated by FACS under the dynamic condition and the social dysfunction index was negative and significant, $r$ $=-.51, p=.034$ (Fig. 4), indicating that individuals with ASD who exhibited fewer mimicry responses to dynamic facial expressions had more severe symptoms in the social domain. The correlation between the frequency of facial mimicry evaluated by FACES under the dynamic condition and the social dysfunction index also revealed a non-significant trend, $r=-.39, p=.096$. Neither FACS-based nor FACES-based facial mimicry under the static condition was significantly correlated with the social dysfunction index (FACS: $r=.06, p=.421$; FACES: $r=-.11$, $p=.356)$.

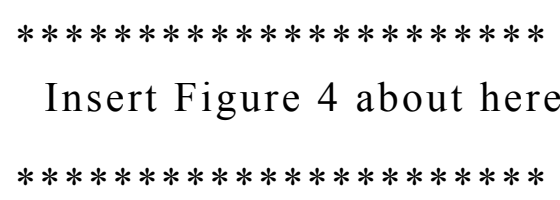

Relationships between facial mimicry and non-social dysfunction 
Impaired overt facial mimicry in ASD

and between intentional imitation and social dysfunction were also submitted to exploratory analysis. The non-social dysfunction index was not significantly correlated with any of the frequencies of facial mimicry under the dynamic condition (FACS: $r=-.16, p=.573$; FACES: $r=-.24$, $p=.412$ ) and the static condition (FACS: $r=.00, p=.997$; FACES: $r=-$. $09, p=.737)$. The frequencies of intentional imitation showed no significant correlation with the social dysfunction index (FACS-dynamic: $r=-.16, p=.582 ;$ FACS-static: $r=-.39, p=.166$; FACES-static: $r=-.17$, $p=.563)$, except that FACES-based intentional imitation under the dynamic condition was significantly and negatively correlated with the social dysfunction index, $r=-.57, p=.041$.

\section{Discussion}

The results in the control group revealed that overt facial muscular activities and emotional expressions congruent with the presented facial expressions were more frequently elicited under the dynamic than under the static presentation condition. These results are consistent with the findings of a previous study (Sato and Yoshikawa 2007) and indicate that dynamic facial expressions evidently induce overt facial mimicry in typically developing individuals. 
Impaired overt facial mimicry in ASD

More importantly, the ASD group exhibited less frequent facial muscle activity and emotional expressions congruent with others' facial expressions than did the control group. These findings are not explained by differences in participants' attention to the stimuli, as indicated by the absence of significant differences in attention between the groups.

Therefore, the ASD group had reduced overt facial mimicry. This result is consistent with the findings of previous realistic studies (e.g., Yirmiya et al. 1989). However, those studies could not exclude the possibility that environmental stimuli other than facial expressions and the contexts of social interactions influenced facial actions. Additionally, the reduction in facial mimicry is in part consistent with previous EMG studies using well-controlled conditions in which stimuli other than facial expressions were removed, and the effects of context were excluded (i.e., conditions under which the effects of factors other than facial expressions on facial actions could be ignored) (e.g., McIntosh et al. 2006). However, EMG studies may not necessarily measure overt facial expressions. In this study, we used well-controlled conditions and measured overt facial expressions. Therefore, to our knowledge, this is the first strict study to demonstrate impairments in externally observable facial mimicry in ASD.

Our results also revealed that reduced overt facial mimicry in ASD 
Impaired overt facial mimicry in ASD

was evident when facial expression stimuli were presented dynamically. Consistent with this finding, previous studies have suggested that dynamic presentations of facial stimuli more clearly elicited atypical behavioral patterns in individuals with ASD than did static presentations (Kessels et al. 2010; Tardif et a1. 2007; Uono et a1. 2009). The result is also consistent with several neuroimaging studies reporting that individuals with ASD exhibited reduced activation in response to dynamic facial expressions compared with the control group (Pelphrey et al. 2007; Sato et a1. 2012) in some brain regions that are thought to be the neural bases of facial mimicry (Likowski et al. 2012). These data suggest that the impairment in social interactions in $\mathrm{ASD}$, including reduced overt facial mimicry, is more evident in response to dynamic facial expressions.

Furthermore, our results regarding ASD group revealed that reduced overt facial mimicry in response to dynamic facial expressions was related to the severity of social dysfunction, but not to non-social dysfunction. This finding suggests that the impairment in overt facial mimicry in individuals with ASD plays an important role in their social dysfunction in the real world. Although the exact mechanism of this relationship requires further investigation, studies involving typically 
Impaired overt facial mimicry in ASD

developing individuals provide clues regarding this issue. Some studies have revealed that facial mimicry facilitates reciprocal social interactions, such as the establishment of rapport and the development of empathy (e.g., Cappella 1993; for a review, see Hess et al. 1999). Based on these data, we speculate that individuals with ASD may have difficulty establishing rapport and developing empathy with partners during communication partly because of impaired overt facial mimicry, which can lead to impairments in reciprocal social relationships. Other studies have also found that facial mimicry affects some intra-individual social cognitive processes, such as recognition of emotion in response to others' facial expressions (Künecke et al. 2014; Sato et al. 2013). Such data suggest the possibility that reduced facial mimicry in individuals with ASD may lead to difficulty understanding another person's emotions, which can result in socially inappropriate behaviors. However, it must be noted that the psychological processes related to facial mimicry, even among typically developing individuals, remain controversial (e.g., Blairy et al. 1999), so further studies are necessary to specify the mechanisms linking impaired facial mimicry and social dysfunction in individuals with ASD.

In contrast to their impaired spontaneous facial mimicry, the ASD group had less evident problems with the intentional imitation of facial 
Impaired overt facial mimicry in ASD

expressions. This result is consistent with previous findings indicating normal voluntary imitation but impaired spontaneous mimicry in response to others' facial expressions in those with ASD (McIntosh et al. 2006; Oberman et al. 2009). These results suggest that impairments in facial mimicry in response to dynamic facial expressions in individuals with ASD are not due to an inability to produce facial expressions.

Additionally, these results may have implications for clinical intervention. Several previous studies involving typically developing individuals have demonstrated that intentional motor imitation of partners in social interactions enhanced rapport with and empathy in the partners (e.g., Maurer and Tindall 1983; cf. Hess and Blairy 2001). Other studies have also reported that intentional facial imitation facilitates sharing of emotions in response to facial expressions (e.g., Dimberg and Söderkvist 2011). Given that individuals with ASD are capable of intentional facial imitation, the use of intentional imitation of others' facial expressions may improve the ability of these individuals to establish rapport with their communication partners and to feel empathy for their partners. However, note that our results showed a relationship between reduced intentional imitation in response to dynamic facial expressions and the severity of social dysfunction in ASD. Further investigation of the 
Impaired overt facial mimicry in ASD

impairment in intentional facial imitation in individuals with ASD and its functional significance would be valuable.

Some possible limitations of this study should be noted. First, the frequency of congruent facial reactions was low even under the dynamic presentation condition in typically developing controls. The results suggest that the current stimuli were too weak to elicit mimicry responses. However, comparable data revealing a low frequency of overt facial mimicry in response to dynamic facial expressions were reported in previous studies with adults (Sato and Yoshikawa 2007) and infants (Field et al. 1982). Some previous EMG studies have also reported that facial muscle responses detected in EMG signals are normally invisible when the stimuli are photographs (e.g., Cacioppo et al. 1992). These data suggest that congruent facial muscle activity in response to facial expressions is generally subtle and that overt facial mimicry occurs with relatively low frequency. However, such low-frequency facial mimicry plays an important role in social interactions among typically developing participants (cf. Hess et al. 1999), and our data suggest that it is impaired in individuals with ASD.

Second, we targeted only individuals with ASD who had relatively milder autistic traits, that is, individuals with Asperger's disorder and the 
Impaired overt facial mimicry in ASD

milder-symptom subgroup of PDD-NOS. Hence, it remains unknown whether impaired overt facial mimicry is also present in individuals with more severe ASD symptoms (e.g., autistic disorder). Our correlation analyses revealed that reduced facial mimicry was related to more severe ASD symptoms. Based on these data, we speculate that individuals with more severe ASD symptoms may exhibit impaired overt facial mimicry and that this may be more obvious than the impairment in individuals with milder ASD symptoms. It would be interesting to compare impairments in overt facial mimicry across broad autistic subtypes in future studies.

In conclusion, we demonstrated that individuals with milder ASD symptoms exhibit reduced overt facial mimicry in response to dynamic facial expressions and that the reduced overt facial mimicry was related to the severity of social dysfunction. This suggests that impairments in overt facial mimicry in those with ASD may underlie difficulties in real-world social interactions. 
Impaired overt facial mimicry in ASD

\section{Role of the funding source}

This study was supported by funds from the JSPS Funding Program for Next Generation World-Leading Researchers and the Organization for Promoting Research in Developmental Disorders.

\section{Acknowledgement}

The authors thank all participants for their participation in the experiment and Ms. Minemoto and Ms. Yokoyama for their technical assistance. 
Impaired overt facial mimicry in ASD

\section{References}

American Psychiatric Association. (2000). Diagnostic and statistical manual of mental disorders (4th edition, text revision). Washington, DC: American Psychiatric Association.

Beall, P. M., Moody, E. J., McIntosh, D. N., Hepburn, S. L., \& Reed, C. L. (2008). Rapid facial reactions to emotional facial expressions in typically developing children and children with autism spectrum disorder. Journal of Experimental Child Psychology, 101(3), $206-223$.

Blairy, S., Herrera, P., \& Hess, U. (1999). Mimicry and the judgment of emotional facial expressions. Journal of Nonverbal Behavior, 23, $5-41$.

Cacioppo, J. T., Bush, L. K., \& Tassinary, L. G. (1992). Microexpressive facial actions as a function of affective stimuli: Replication and extension. Personality and Social Psychology Bulletin, 18, 515-526.

Cacioppo, J. T., Petty, R. E., Losch, M. E., \& Kim, H. S. (1986).

Electromyographic activity over facial muscle regions can differentiate the valence and intensity of affective reactions. Journal of Personality and Social Psychology, 50, 260-268.

Cappella, J. N. (1993). The facial feedback hypothesis in human 
Impaired overt facial mimicry in ASD

interaction: Review and speculation. Journal of Language and Social Psychology, 12, 13-29.

De Seta, E., Bertoli, G., De Seta, D., Covelli, E., \& Filipo, R. (2010).

New development in intraoperative video monitoring of facial nerve:

a pilot study. Otology \& Neurotology, 31, 1498-1502.

Dimberg, U., \& Söderkvist, S. (2011). The voluntary facial action technique: A method to test the facial feedback hypothesis. Journal of Nonverbal Behavior, 35, 17-33.

Ekman, P., \& Friesen, W. (1978). Facial action coding system: A technique for the measurement of facial movement. Palo Alto, CA: Consulting Psychologist Press.

Ekman, P., Rosenberg, E., \& Hager, J. (1998). Facial action coding system affect interpretation database (FACSAID), http://nirc.com/Expression/FACSAID/facsaid.html, July 1998

Field, T., Woodson, R., Greenberg, R., \& Cohen, D. (1982).

Discrimination and imitation of facial expressions by neonates. Science, 218, 179-181. 
Impaired overt facial mimicry in ASD

Filipo, R., Pichi, B., Bertoli, G. A., \& De Seta, E. (2002). Video-Based System for Intraoperative Facial Nerve Monitoring: Comparison with Electromyography. Otology \& Neurotology, 23, 594-597.

Goodwin, C. (2002). Time in action. Current Anthropology, 43, 19-35.

Hess, U., \& Blairy, S. (2001). Facial mimicry and emotional contagion to dynamic emotional facial expressions and their influence on decoding accuracy. International Journal of Psychophysiology, 40, $129-141$.

Hess, U., \& Fischer, A. (2013). Emotional mimicry as social regulation. Personality and Social Psychology Review, 17, 142-157.

Kessels, R. P. C., Spee, P., \& Hendriks, A. W. (2010). Perception of dynamic facial emotional expressions in adolescents with autism spectrum disorders (ASD). Translational Neuroscience, 1, 228-232.

Koyama, T., Tachimori, H., Osada, H., Takeda, T., \& Kurita, H. (2007). Cognitive and symptom profiles in Asperger's syndrome and high-functioning autism. Psychiatry and Clinical Neurosciences, $61(1), 99-104$.

Kilts, C. D., Egan, G., Gideon, D.A., Ely, T. D., \& Hoffman, J. M. (2003). Dissociable neural pathways are involved in the recognition of 
Impaired overt facial mimicry in ASD

emotion in static and dynamic facial expressions. Neuroimage, 18, $156-168$

Kirk, R. E. (1995). Experimental design: Procedures for the behavioral sciences (3rd ed.). Pacific Grove, CA: Brooks/Cole.

Kring, A.M., \& Sloan, D.M. (2007). The Facial Expression Coding System (FACES): Development, validation, and utility. Psychological Assessment, 19, 210-224.

Künecke, J., Hildebrandt, A., Recio, G., Sommer, W., \& Wilhelm, O. (2014). Facial EMG responses to emotional expressions are related to emotion perception ability. PLoS One, 9, e84053.

Kurita, H., Miyake, Y., \& Katsuno, K. (1989). Reliability and validity of the Childhood Autism Rating Scale-Tokyo version (CARS-TV). Journal of Autism and developmental Disorders, 19(3), 389-396.

LaBar, K. S., Crupain, M. J., Voyvodic, J. T., \& McCarthy, G. (2003). Dynamic perception of facial affect and identity in the human brain. Cerebral Cortex, 13, 1023-1033.

Likowski, K.U., Mühlberger, A., Gerdes, A. B. M., Wieser, M. J., Pauli, P., \& Weyers, P. (2012). Facial mimicry and the mirror neuron system: Simultaneous acquisition of facial electromyography and 
Impaired overt facial mimicry in ASD

functional magnetic resonance imaging. Frontiers in Human

Neuroscience, doi: 10.3389/fnhum.2012.00214

Magyar, C. I., \& Pandolfi, V. (2007). Factor structure evaluation of the Childhood Autism Rating Scale. Journal of Autism and Developmental Disorders, 37, 1787-1794.

Maurer, R. E., \& Tindall, J. H. (1983). Effect of postural congruence on client's perception of counselor empathy. Journal of Counseling Psychology, 30, 158-163.

McIntosh, D. N., Reichmann-Decker A, Winkielman, P., \& Wilbaarger, J. L. (2006). When the social mirror breaks: Deficits in automatic, but not voluntary, mimicry of emotional facial expressions in autism. Developmental Science, 9, 295-302.

Nakano, T., Yamamoto, Y., Kitajo, K., Takahashi, T., \& Kitazawa, S. Synchronization of spontaneous eyeblinks while viewing video stories. (2009). Proceedings. Biological Sciences/The Royal Society, $276,3635-3644$.

Oberman, L. M., Winkielman, P., \& Ramachandran, V. S. (2009). Slow echo: Facial EMG evidence for the delay of spontaneous, but not voluntary, emotional mimicry in children with autism spectrum 
Impaired overt facial mimicry in ASD

disorders. Developmental Science, 12(4), 510-520.

Oldfield, R. C. (1971). The assessment and analysis of handedness: The Edinburgh inventory. Neuropsychologia, 9, 97-113.

Pelphrey, K. A., Morris, J. P., McCarthy, G., \& Labar, K.S. (2007).

Perception of dynamic changes in facial affect and identity in autism.

Social Cognitive and Affective Neuroscience, 2, 140-149.

Sato, W., Fujimura, T., \& Suzuki, N. (2008). Enhanced facial EMG activity in response to dynamic facial expressions. International Journal of Psychophysiology, 70(1), 70-74.

Sato, W., Fujimura, T., Kochiyama, T., \& Suzuki, N. (2013).

Relationships among facial mimicry, emotional experience, and emotion recognition. PLoS ONE, 8, e57889.

Sato, W., Kochiyama, T., Yoshikawa, S., Naito, E., \& Matsumura, M. (2004). Enhanced neural activity in response to dynamic facial expressions of emotion: An fMRI study. Cognitive Brain Research, $20,81-91$.

Sato, W., Toichi, M., Uono, S., \& Kochiyama, T. (2012). Impaired social brain network for processing dynamic facial expressions in autism spectrum disorders. BMC Neuroscience, doi:

10.1186/1471-2202-13-99 
Impaired overt facial mimicry in ASD

Sato, W., \& Yoshikawa, S. (2007). Spontaneous facial mimicry in response to dynamic facial expressions. Cognition, 104, 1-18.

Schopler, E, Reichler, R. J., \& Renner, B. R. (1986). The Childhood Autism Rating Scale (CARS): For diagnostic screening and classification of autism. New York, NY: Irvington.

Stel, M., van den Heuvel, C., \& Smeets R. C. (2008). Facial feedback mechanisms in autistic spectrum disorders. Journal of Autism and Developmental Disorders, 38(7), 1250-1258.

Tabachnick, B. G., \& Fidell, L. S. (2001). Computer-assisted research design and analysis. Boston, MA: Allyn and Bacon.

Tachimori, H., Osada, H., \& Kurita, H. (2003). Childhood Autism Rating Scale - Tokyo Version for screening pervasive developmental disorders. Psychiatry and Clinical Neurosciences, 57(1), 113-118.

Tardif, C., Lainé, F., Rodriguez, M., \& Gepner, B. (2007). Slowing down presentation of facial movements and vocal sounds enhances facial expression recognition and induces facial-vocal imitation in children with autism. Journal of Autism and Developmental Disorders, 37, 1469-1484.

Uono, S., Sato, W., \& Toichi, M. (2009). Dynamic fearful gaze does not 
Impaired overt facial mimicry in ASD

enhance attention orienting in individuals with Asperger's disorder. Brain and Cognition, 71, 229-233.

Uono, S., Sato, W., \& Toichi, M. (2011). The specific impairment of fearful expression recognition and its atypical development in pervasive developmental disorder. Social Neuroscience, 6, 452-463.

Weyers, P., Mühlberger, A., Hefele, C., \& Pauli. P. (2006).

Electromyographic responses to static and dynamic avatar emotional facial expressions. Psychophysiology, 43(5), 450-453.

Yirmiya, N., Kasari, C., Sigman, M., \& Mundy, P. (1989). Facial expressions of affect in autistic, mentally retarded and normal children. Journal of Child Psychology and Psychiatry, and Allied Disciplines, 30(5), 725-735. 
Impaired overt facial mimicry in ASD

\section{Figure Captions}

Figure 1. Examples of participants' facial reactions. Brow lowering (i.e. Action Unit (AU) 4) in response to an angry expression (a) and lip-corner pulling (i.e., AU 12) in response to a happy expression (b) by one of the typically developing controls are shown.

Figure 2. (a) Mean (with $S E$ ) percent occurrence of Action Unit (AU) 4 (left) and AU 12 (right) in the facial mimicry task. (b) Mean (with $S E$ ) percent occurrence of negative (left) and positive (right) valence in the facial mimicry task. $* * * p<.005 ; * p<.05$.

Figure 3. (a) Mean (with $S E$ ) percent occurrence of Action Unit (AU) 4 (left) and AU 12 (right) in the intentional imitation task. (b) Mean (with $S E)$ percent occurrence of negative (left) and positive (right) valence in the intentional imitation task.

Figure 4. The scatter plot of relationship between the degree of social impairments evaluated by the CARS and the frequency of facial mimicry 
Impaired overt facial mimicry in ASD

evaluated by Facial Action Coding System (FACS) under the dynamic condition in the ASD group. The regression lines are also plotted. The reduced facial mimicry in response to dynamic facial expressions, evaluated by FACS, predicts severe social impairments. 
(a)

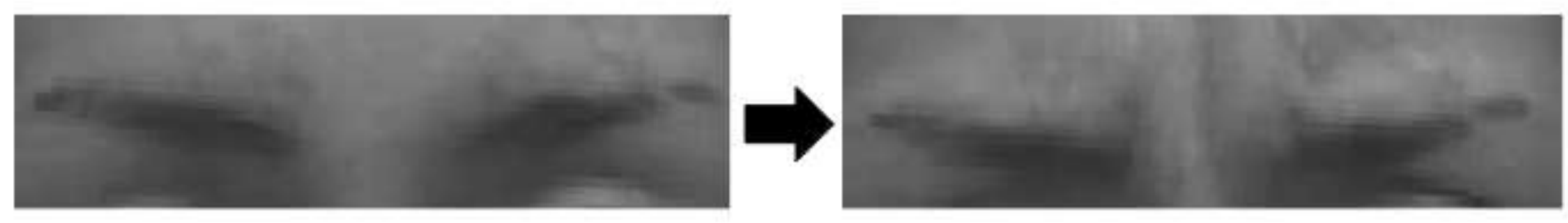

(b)
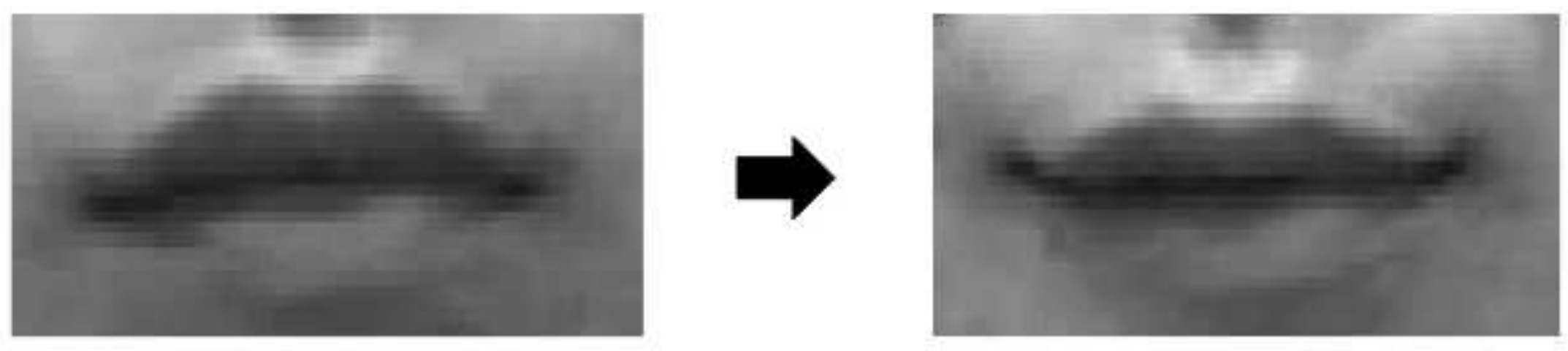
(a)

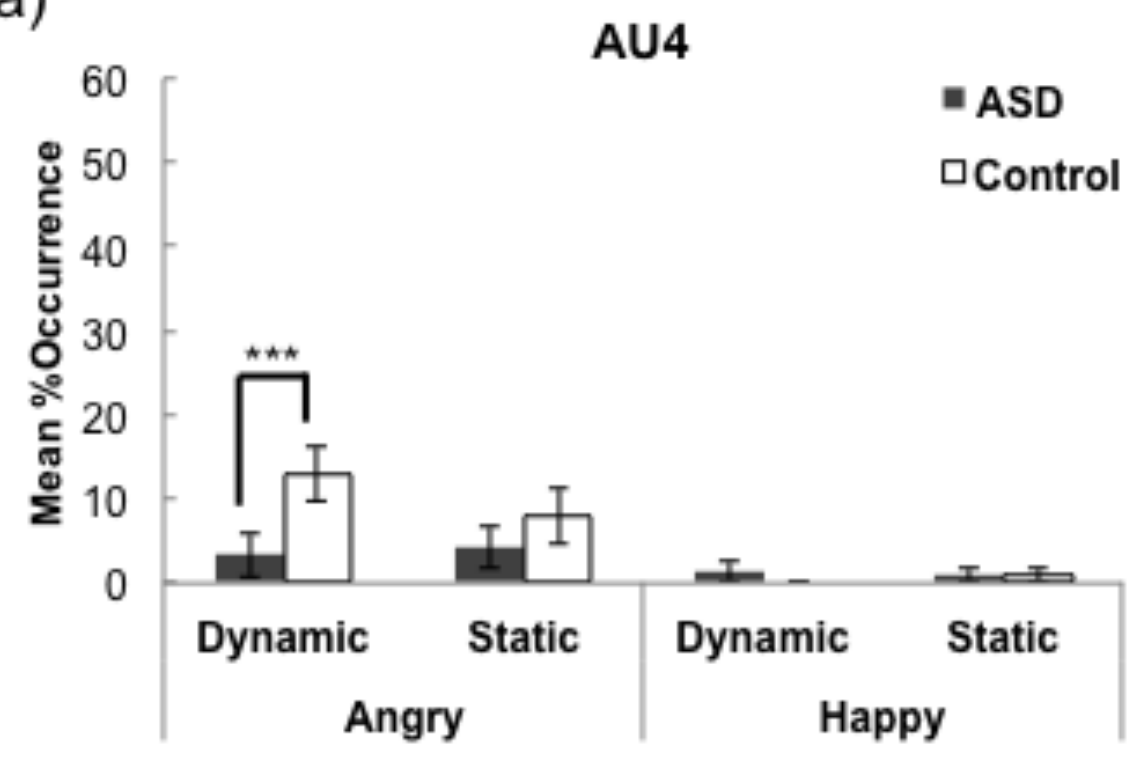

(b)

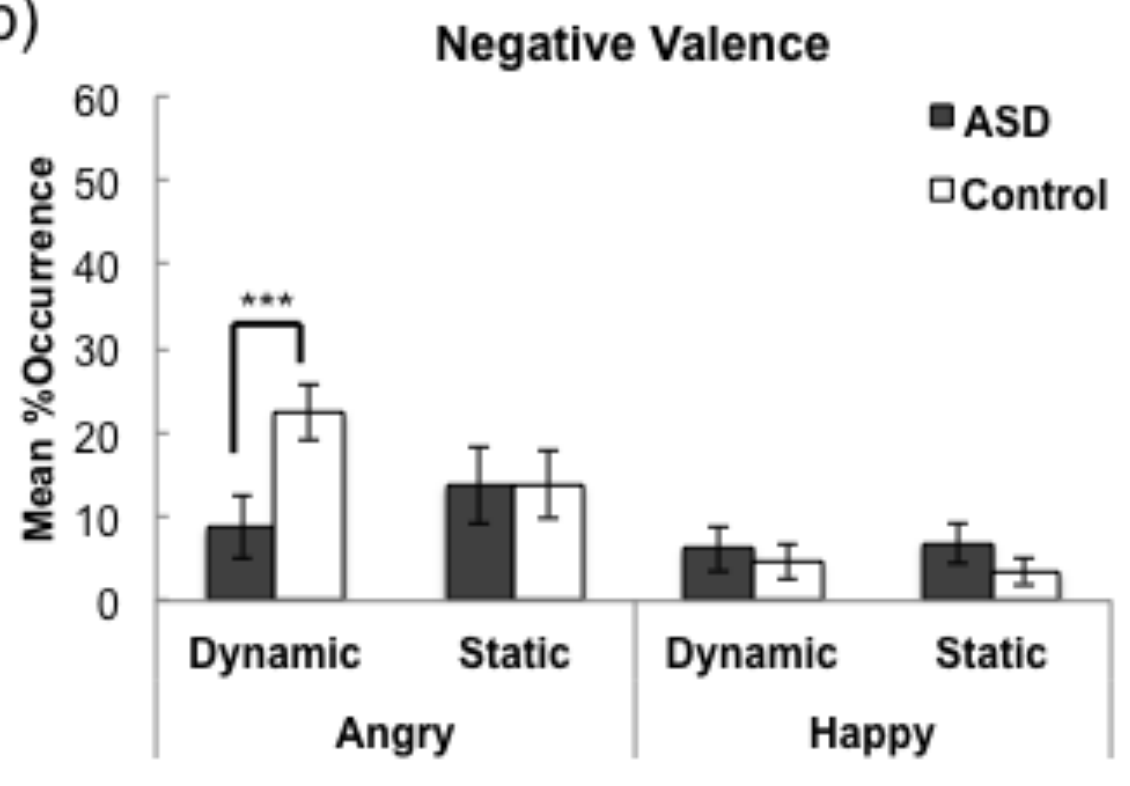

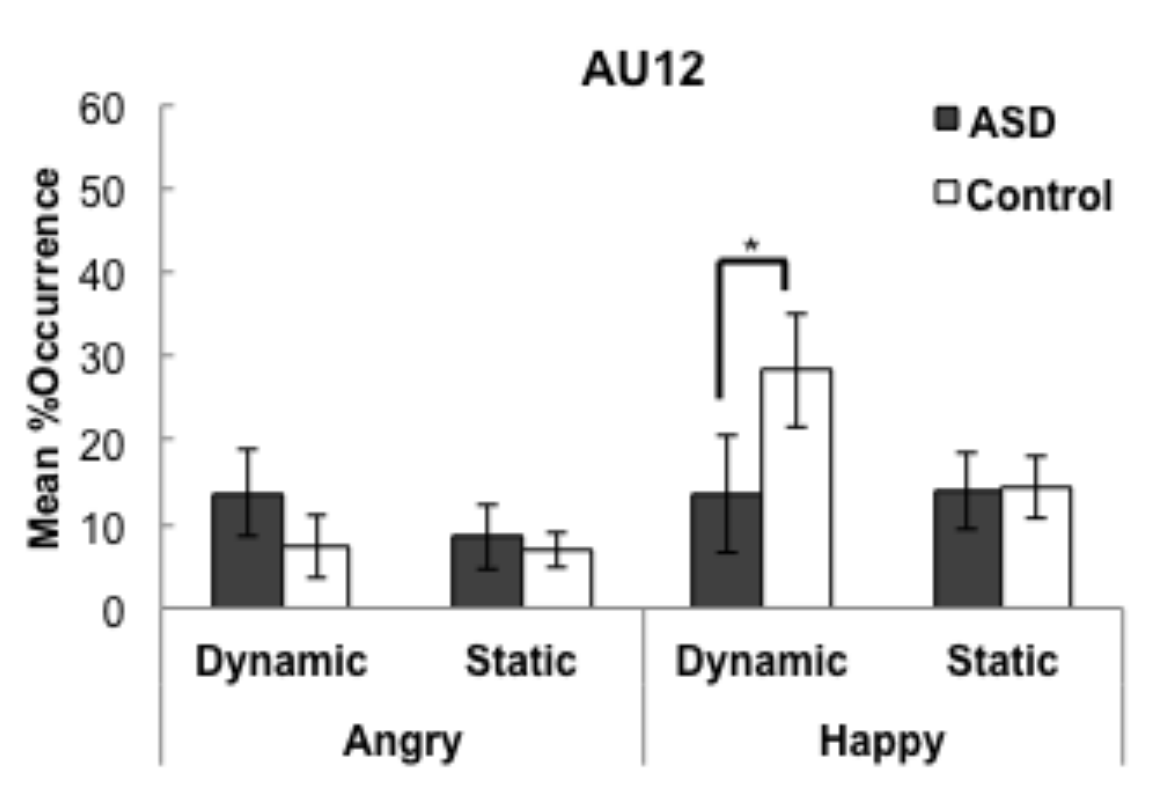

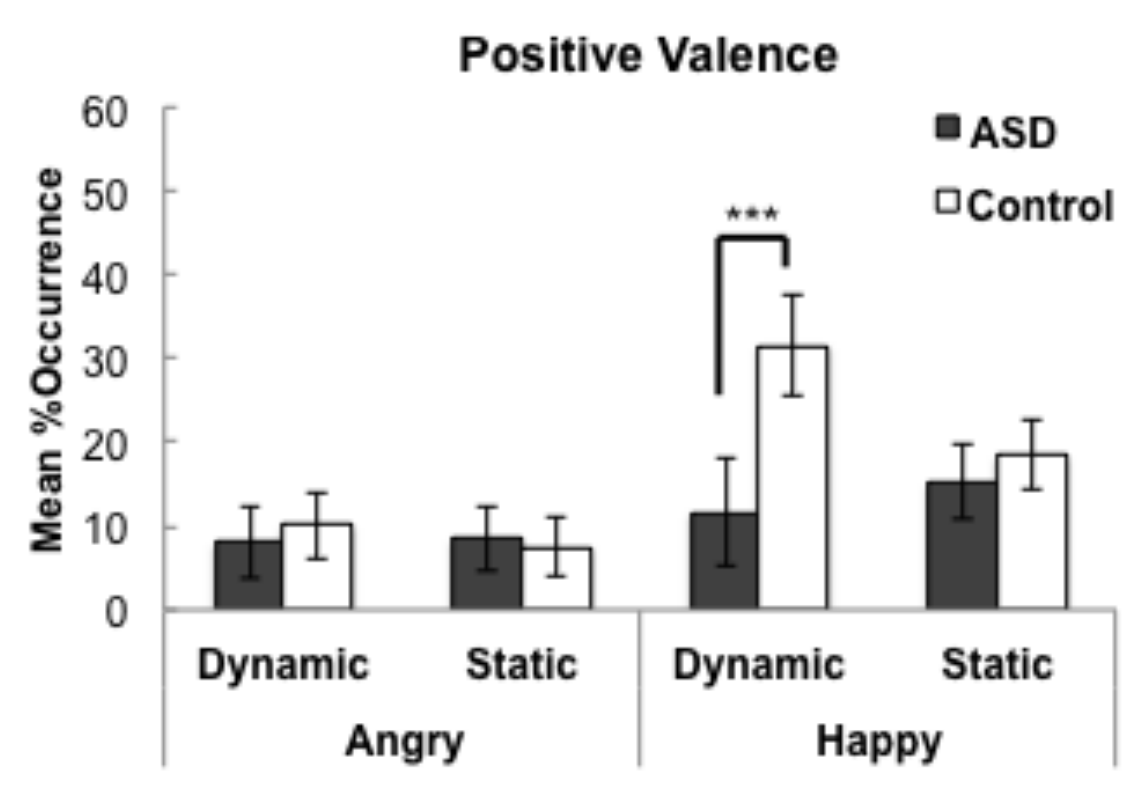


(a)

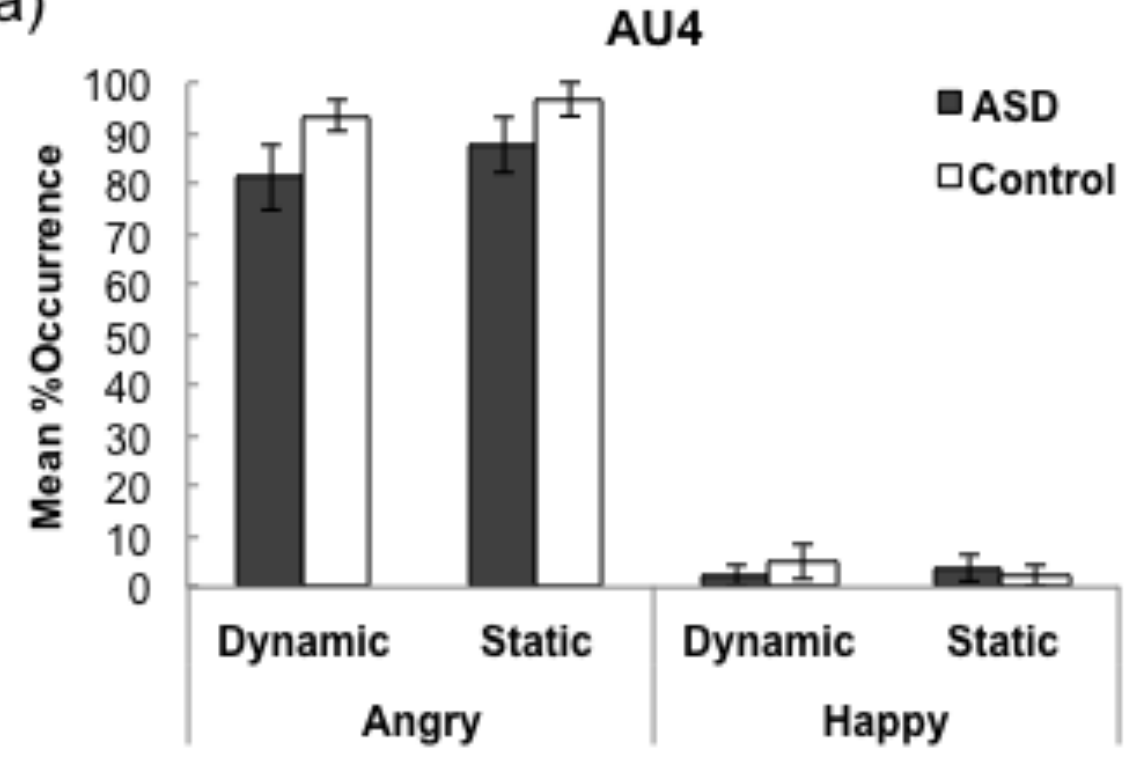

(b)

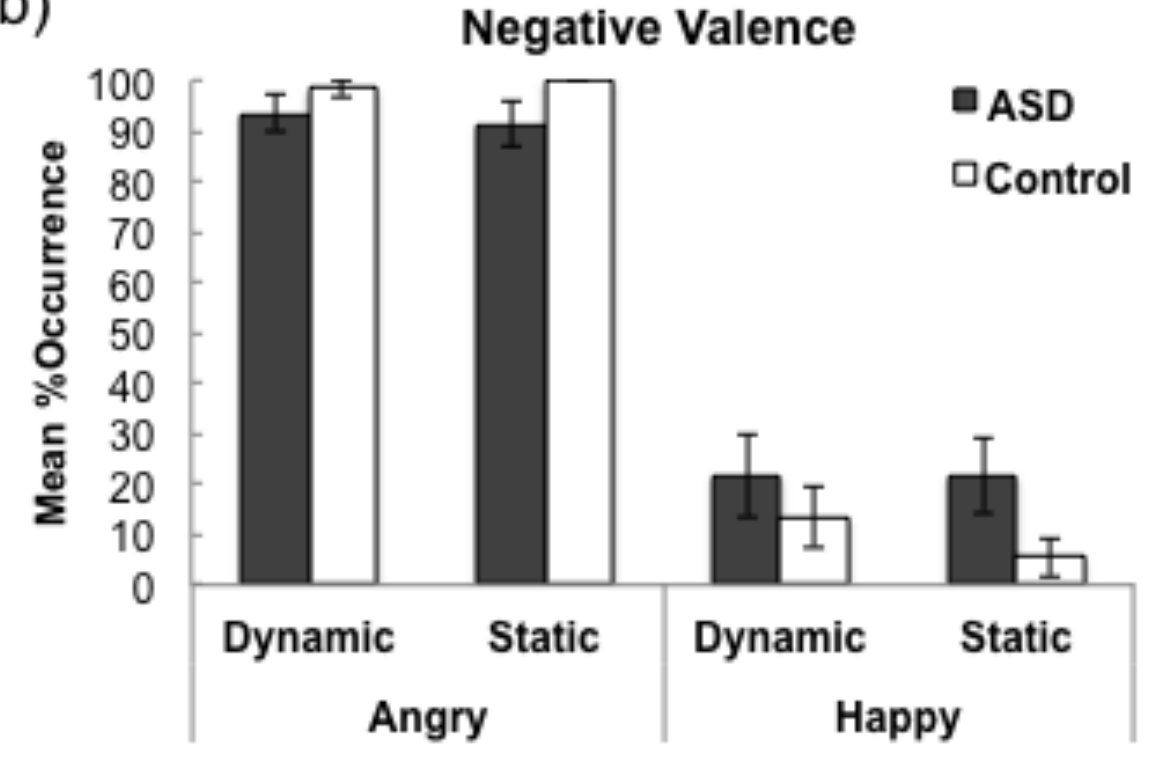

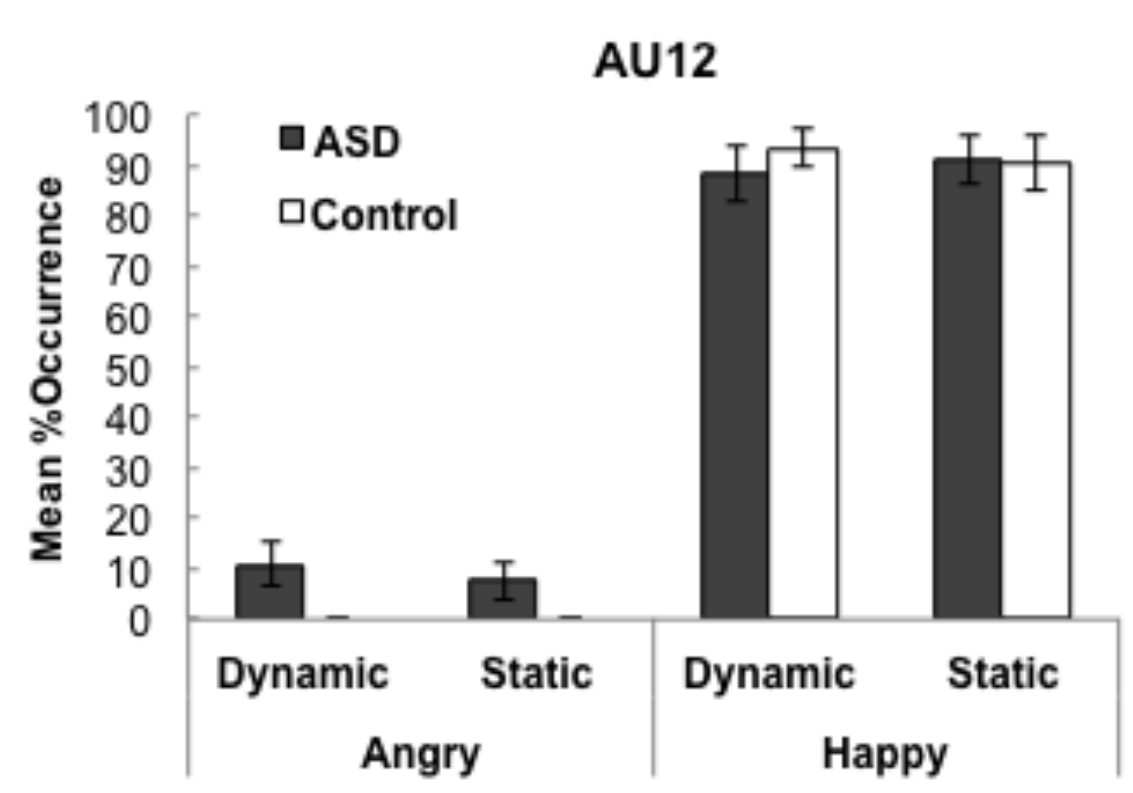

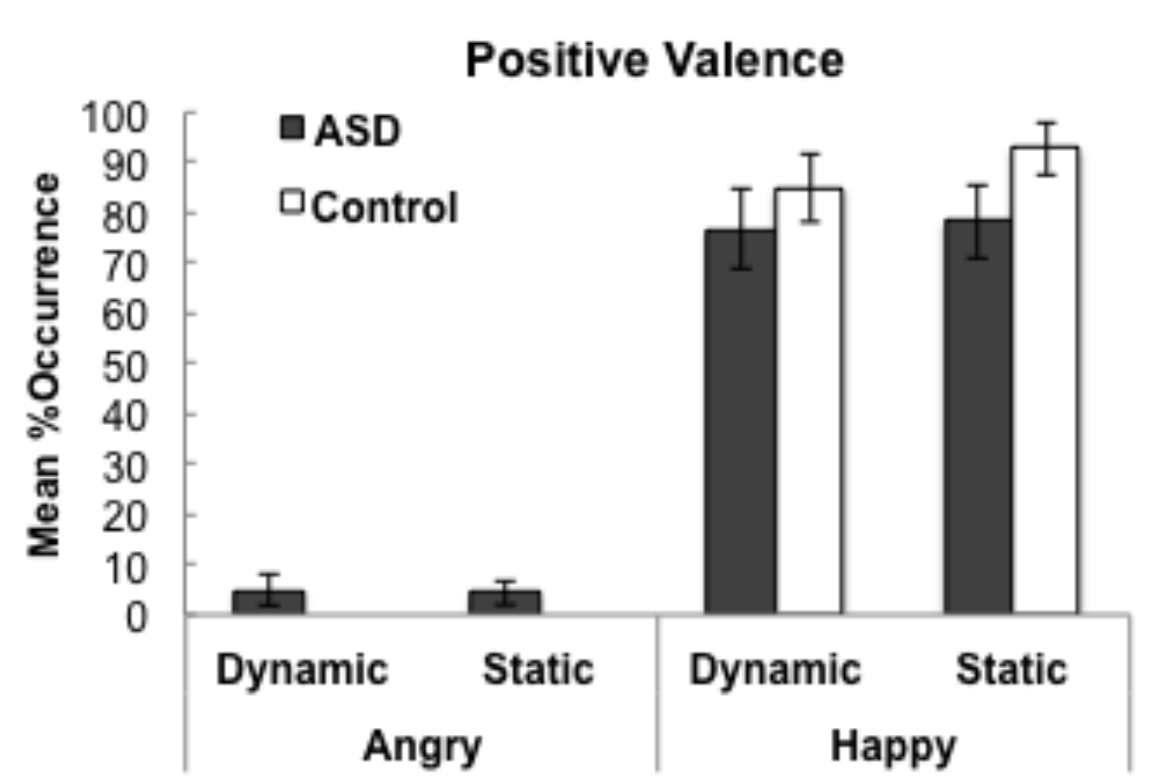




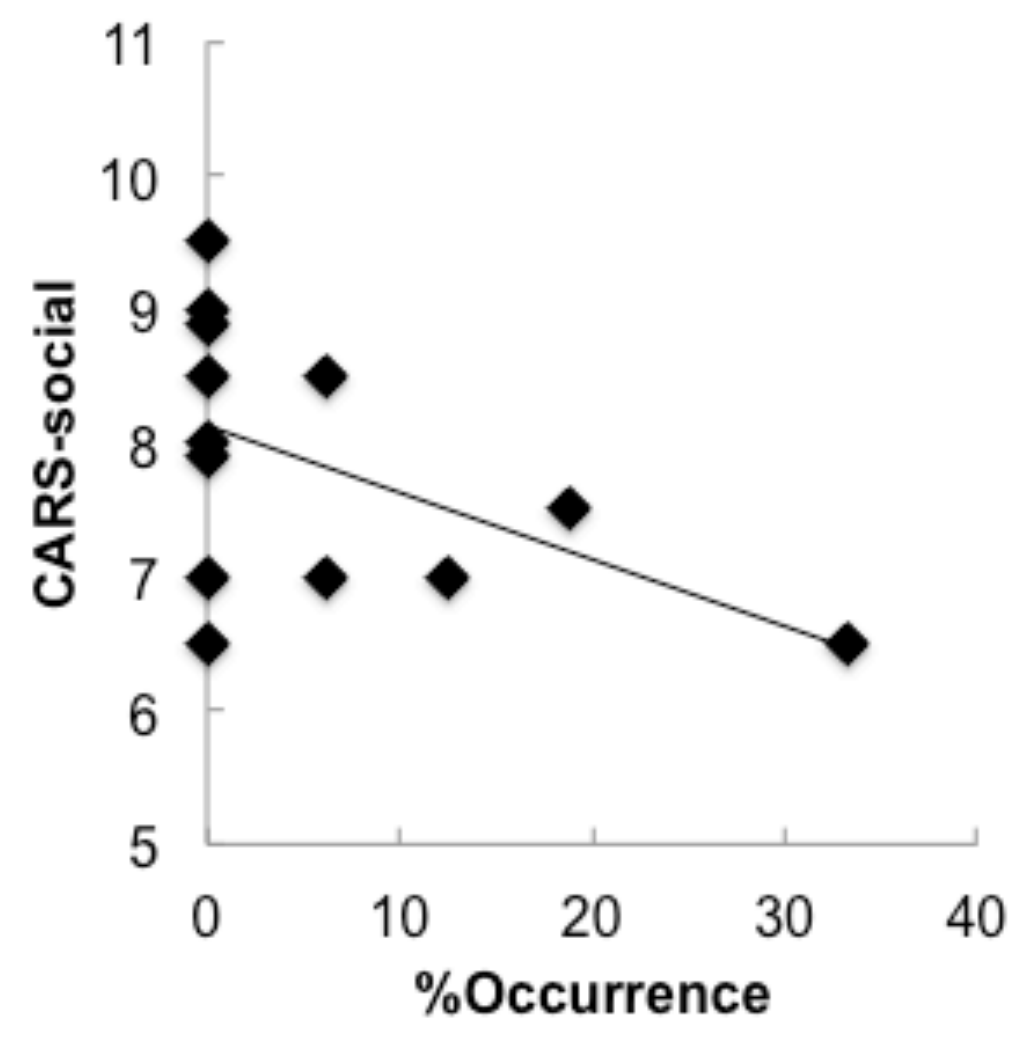


Impaired overt facial mimicry in ASD

\section{Supplementary material}

Online Resource 1. Action Units of the Facial Action Coding System (FACS) (Ekman and Friesen, 1978)

\begin{tabular}{|c|c|c|}
\hline Action Unit & Description in FACS & Muscular basis \\
\hline 1 & Inner Brow Raiser & Frontalis (pars medialis) \\
\hline 2 & Outer Brow Raiser & Frontalis (pars lateralis) \\
\hline 4 & Brow Lowerer & $\begin{array}{c}\text { Depressor glabellae, Depressor supercilii, } \\
\text { Corrugator supercilii }\end{array}$ \\
\hline 5 & Upper Lid Raiser & Levator palpebrae superioris \\
\hline 6 & $\begin{array}{l}\text { Cheek Raiser and Lid } \\
\text { Compressor }\end{array}$ & Orbicularis oculi (pars orbitalis) \\
\hline 7 & Lid Tightener & Orbicularis oculi (pars palpebralis) \\
\hline 9 & Nose Wrinkler & Levator labii superioris alaeque nasi \\
\hline 10 & Upper Lip Raiser & Levator labii superioris \\
\hline 11 & Nasolabial Furrow Deepener & Zygomaticus minor \\
\hline 12 & Lip Corner Puller & Zygomaticus major \\
\hline 13 & Sharp Lip Puller & Levator anguli oris \\
\hline
\end{tabular}

(Continued) 
Impaired overt facial mimicry in ASD

\begin{tabular}{|c|c|c|}
\hline Action Unit & Description in FACS & Muscular basis \\
\hline 14 & Dimpler & Buccinator \\
\hline 15 & Lip Corner Depressor & Depressor anguli oris \\
\hline 16 & Lower Lip Depressor & Depressor labii inferioris \\
\hline 17 & Chin Raiser & Mentalis \\
\hline 18 & Lip Pucker & $\begin{array}{c}\text { Incisivii labii superioris and Incisivii labii } \\
\text { inferioris }\end{array}$ \\
\hline 20 & Lip Stretcher & Risorius $w /$ Platysma \\
\hline 22 & Lip Funneler & Orbicularis oris \\
\hline 23 & Lip Tightener & Orbicularis oris \\
\hline 24 & Lip Presser & Orbicularis oris \\
\hline 25 & Lips Part & Depressor labii inferioris, or relaxation of \\
\hline & & Mentalis or Orbicularis oris \\
\hline 26 & Jaw Drop & Masseter, relaxed Temporalis and Internal \\
\hline & & Pterygoid \\
\hline 27 & Mouth Stretch & Pterygoids, Digastric \\
\hline 28 & Lip Suck & Orbicularis oris \\
\hline 41 & Lid droop & $\begin{array}{c}\text { Relaxation of Levator palpebrae } \\
\text { superioris }\end{array}$ \\
\hline
\end{tabular}

(Continued) 
Impaired overt facial mimicry in ASD

\begin{tabular}{|c|c|c|}
\hline Action Unit & Description in FACS & Muscular basis \\
\hline \multirow[t]{2}{*}{42} & Slit & Orbicularis oculi \\
\hline & & Relaxation of Levator palpebrae \\
\hline \multirow[t]{2}{*}{43} & Eye Closure & superioris; Orbicularis oculi (pars \\
\hline & & palpebralis) \\
\hline \multirow[t]{2}{*}{44} & Squint & Orbicularis oculi (pars palpebralis) \\
\hline & & Relaxation of Levator palpebrae \\
\hline \multirow[t]{3}{*}{45} & Blink & superioris; Orbicularis oculi (pars \\
\hline & & palpebralis) \\
\hline & & Relaxation of Levator palpebrae \\
\hline \multirow[t]{2}{*}{46} & Wink & superioris; Orbicularis oculi (pars \\
\hline & & palpebralis) \\
\hline
\end{tabular}

